\title{
Prevalence of financial conflicts of interest among panel members producing clinical practice guidelines in Canada and United States: cross sectional study
}

In this research paper by Jennifer Neuman and colleagues (BMJ 2011;343:d5621, doi:10.1136/bmj.d5621) the second sentence of the second paragraph under "Prevalence of financial COI" should have read: "All eight [not 73] panel members received speakers' fees, honorariums, or employee/adviser/consultancy payments or held stock ownership." In addition, the author of reference 27 is IMS [not "Institute of Medicine"].

Cite this as: BMJ 2011;343:d7063

๑ BMJ Publishing Group Ltd 2011 